\title{
Möglichkeiten der biochemischen Differenzierung von Reizergüssen am Kniegelenk
}

\author{
M. D. Dingerkus ${ }^{\prime}$, M. Jochum ${ }^{2}$, H. Fritz' ${ }^{2}$, P. Bernett ${ }^{\prime}$
}

'Sporttraumatologie mit Klinik und Poliklinik für Sportverletzungen, Klinikum Rechts der Isar, Technische Universität München

${ }^{2}$ Institut für Klinische Chemie und Klinische Biochemie der Chirurgischen Universitätsklinik, Innenstadt, München

\section{Zusammenfassung}

1. Reizerguß-Differenzierung: Posttraumatische Reizergüsse (frische Meniskus- oder Kapsel-Band-Läsion) zeigten eine stark erhöhte alkalische Phosphatasenaktivität, während zellarme und zellreiche postoperative Reizergüsse einen im Vergleich zum Gesamtprotein verhältnismäßig hohen $\mathrm{C} 3 \mathrm{c}$-Wert aufwiesen. In Punktaten von Patienten mit Chondropathia patellae und primärer Synoviareizung (kein Trauma, kein Postoperativzustand) wurden die geringsten Parameterwerte der gesamten Untersuchung gefunden.

2. Therapie-Entscheidungshilfe: Über eine Bestimmung des sensiblen Entzündungsparameter PMN-Elastase $\left(\mathrm{E}-\mathrm{a}_{1} \mathrm{PI}\right)$ kann etwa bei einem Knorpelschaden mit geringem Entzündungsgrad (E$a_{1} \mathrm{Pl}<300-500 \mathrm{ng} / \mathrm{ml}$ ) sofort eine knorpelprotektive Therapie eingeleitet werden, während bei starkem Entzündungsgrad $\left(-\mathrm{a}_{4} \mathrm{Pl}>500-1000 \mathrm{ng} / \mathrm{ml}\right)$ zunächst Antiphlogistika einzusetzen sind.

3. Therapie-Kontrolle: In der Verlaufsbeobachtung von Reizergüssen liefert der Konzentrationsvergleich biochemischer Parameter vor und nach intraartikulärer Applikation von Medikamenten die Möglichkeit der Therapiekontrolle. Da bei zunehmendem Entzündungscharakter und Störung der Blut-SynoviaSchranke die Konzentration der gemessenen biochemischen $\mathrm{Pa}$ rameter (ausgenommen Glukose) in der Synovialflüssigkeit anstieg, sollte bei einer erfolgreich durchgeführten Behandlung eine derartige Konzentrationszunahme nicht oder nur noch in geringem $A$ usma $B$ auftreten.

\section{Possibilities of Biochemical Differentiation of Reac- tion Effusions in the Knee Joint}

1. Differentiation of reaction effusions: Posttraumatic reaction ef fusions (fresh meniscus or capsular ligament lesion) displayed a greatly enhanced alkaline phosphatase activity, whereas postoperative reaction effusions that were poor and rich in cells had a comparatively high $\mathrm{C} 3 \mathrm{c}$ value relative to the total amount of protein. In punctates from patients with patellar chondropathy and primary synovial reaction (no trauma, no postoperative condition) the lowest parameter values of of the entire study were seen.

2. Decision help with regard to therapy: Via determination of the sensitive inflammation parameter PMN elastase $\left(\mathrm{E}-\mathrm{a}_{1} \mathrm{PI}\right)$ it is possible to initiate cartilage-protective therapy directly in case of a cartilage lesion of mild inflammatory character (E-a, $\mathrm{PI}$ $300-500 \mathrm{ng} / \mathrm{ml})$, whereas in case of severe inflammation $\left(\mathrm{E}-\mathrm{a}_{4} \mathrm{Pl}\right.$ $500-1000 \mathrm{ng} / \mathrm{ml}$ ) anti-inflammatory drugs should be employed in the first instance.

3. Therapy control: In the follow-up control of reaction effusions the comparison of concentrations of biochemical parameters before and after intra-articular application of drugs offers a possib. ility of therapy control. Since with increasing inflammatory nature and disturbance of the blood-synovia barrier the concentration of the measured biochemical parameters (with the exception of glucose) increased in the synovial fluid, successful treatment should result in an absence of such an increase in concentration (or, if at all, in an only slight measure).

\section{Einleitung}

Reizergüsse am Kniegelenk werden in der sporttraumatologischen Praxis häufig beobachtet.

Im Hinblick auf mögliche Knorpeldegenerationen als Folge rezidivierender Ergüsse kann die Synoviadiagnostik bedeutsame Entscheidungshilfe für therapeutische Interventionen sein.

\section{Material und Methoden}

Insgesamt wurde die Synovialflüssigkeit von $20 \mathrm{~Pa}$ tienten mit den Diagnosen Chondropathia patellae, posttraumatischer frischer Erguß (Meniskus-/KapselBand-Läsion), Erguß bei Synoviareizung (postoperativ, nicht-postoperativ) sowie bei einer älteren Meniskopathie untersucht (3, Tab. 1).

Sportverletzung - Sportschaden 2 (1987) 86-90

(c) Georg Thieme Verlag. Stuttgart - New York
In 3 Fällen konnte 2 Wochen nach der ersten Punktion ein zweites Mal punktiert werden, so daß eine gewisse Verlaufsbeurteilung der Ergüsse möglich war (3; Tab. 4).

Die biochemische Untersuchungspalette umfaßte mehr als 10 Parameter und reichte von Stoffwechselparametern $(2,5,18)$, Proteinen (18), lysosmalen Enzymen $(1,2,5,17,18)$, Proteinasen und ihren Inhibitoren (7-9) bis hin zu Spaltprodukten von Komplementfak toren $(15,16)$; daneben wurden lösliche proteinhaltige

Tab.1 Auischlüsselung der untersuchten Synovialflusssıgkett nach Patientendiagnosen $(n=20)$

\begin{tabular}{ll}
\hline Chondropathia patellae & $(n=3)$ \\
ältere Meniskopathie & $(n=2)$ \\
frische Meniskusläsion & $(n=5)$ \\
frische Kapsel-Band-Läsion & $(n=1)$ \\
Synoviareizung (Synovialitis) & $(n=1)$ \\
- nicht-postoperativ (primär) & \\
- postoperativ (zellarm, zellreich) & $(n=8)$ \\
\hline
\end{tabular}


Tab.2 Biochemische Untersuchungsparameter in der Synovialflussıgkeit sowie inre Bewertung in der Literatur hinsichtlich Herkunft (Bıldung. Speıcherung) und Aussagekraft fur die Synoviadiagnostik

\begin{tabular}{|c|c|c|c|}
\hline $\begin{array}{l}\text { Bıochemi- } \\
\text { sche } \\
\text { Parameter }\end{array}$ & Herkunft & Aussagekraft & Autor \\
\hline $\begin{array}{l}\text { Glukose. } \\
\text { Laktat. } \\
\text { LDH }\end{array}$ & $\begin{array}{l}\text { Synovial-. } \\
\text { Blut und } \\
\text { Knorpelzellen }\end{array}$ & $\begin{array}{l}\text { Entzündungs- } \\
\text { grad. } \\
\text { Stottwechsel- } \\
\text { situation }\end{array}$ & $\begin{array}{l}\text { Greiling und } \\
\text { Mitarb.,1979 } \\
\text { Binzus. } 1979\end{array}$ \\
\hline $\begin{array}{l}\text { Gesamipro- } \\
\text { tein. } \\
\text { Albumin }\end{array}$ & Serum & $\begin{array}{l}\text { Entzündungs- } \\
\text { grad } \\
\text { Glut-Synovia. } \\
\text { Schranke }\end{array}$ & $\begin{array}{l}\text { Kleesiek, } 1980 \\
\text { Greilıng. } 1979 \\
\text { in 18, S. } 188\end{array}$ \\
\hline $\begin{array}{l}\text { Kompiement- } \\
\text { spaltprodukt } \\
\text { C3c }\end{array}$ & $\begin{array}{l}\text { Serum (C3 im } \\
\text { Knorpel) }\end{array}$ & $\begin{array}{l}\text { Entzündungs- } \\
\text { grad } \\
\text { Blut-Synovia- } \\
\text { Schranke }\end{array}$ & $\begin{array}{l}\text { Otte, } 1976 \\
\text { Rother und } \\
\text { Mitarb., } 1984\end{array}$ \\
\hline $\begin{array}{l}\text { Phosphata- } \\
\text { sen } \\
\text { saure-alkali- } \\
\text { sche- }\end{array}$ & $\begin{array}{l}\text { Blut- und } \\
\text { Knorpelzellen }\end{array}$ & $\begin{array}{l}\text { Entzündungs- } \\
\text { grad } \\
\text { (Knorpeldene. } \\
\text { ratıon?) }\end{array}$ & $\begin{array}{l}\text { Akeson und } \\
\text { Mitarb.. } 1981 \\
\text { Scherak und } \\
\text { Mitarb. } 1979\end{array}$ \\
\hline Lysozym & $\begin{array}{l}\text { Blut- und } \\
\text { Knorpelzellen }\end{array}$ & $\begin{array}{l}\text { Entzündungs- } \\
\text { grad } \\
\text { (Knorpeldege- } \\
\text { neration?) }\end{array}$ & $\begin{array}{l}\text { Akeson und } \\
\text { Mitarb., } 1981 \\
\text { Kuettner und } \\
\text { Mitarb., } 1974\end{array}$ \\
\hline $\begin{array}{l}\text { PMN-Elastase } \\
(\mathrm{E}, \mathrm{PI})\end{array}$ & Granulozyten & $\begin{array}{l}\text { Entzundungs- } \\
\text { grad } \\
\text { (Knorpeldege- } \\
\text { neration) }\end{array}$ & $\begin{array}{l}\text { Gadek und } \\
\text { Mitarb., } 1980 \\
\text { Kleesiek und } \\
\text { Mitarb., } 1984\end{array}$ \\
\hline $\mathrm{a}, \mathrm{PI}$ und $\mathrm{a}_{2} \mathrm{M}$ & $\begin{array}{l}\text { Serum }\left(a_{2} M\right. \\
\text { im Knorpel?) }\end{array}$ & $\begin{array}{l}\text { Blut-Synovia- } \\
\text { Schranke } \\
\text { (Proteinasenın- } \\
\text { hibition) }\end{array}$ & $\begin{array}{l}\text { Kleesiek und } \\
\text { Milarb., } 1982\end{array}$ \\
\hline
\end{tabular}

Abbauprodukte der Knorpelmatrix nachgewiesen (Tab. 2).

Der Nachweis erfolgte teils mit biochemischen Standard-Verfahren, teils mit speziellen biochemischen Methoden wie radialer Immundiffusion (RID-Methode: 13), Enzymimmunoassay $(8,14)$, Immun- und diskontinuierlicher SDS-Elektrophorese* $(11,12 ;$ Tab.3)

\section{Ergebnisse}

Eine primäre Synoviareizung (kein Trauma, kein Postoperativzustand) zeigte die niedrigsten Konzentrationen der gemessenen Parameter aller Diagnosegruppen und dürfte weitgehend den Verhältnissen einer normalen Synovia entsprechen (Abb. 1 a).

Bei Chondropathia patellae fiel der hohe a,M-Wert auf, der nicht permeabilitätsbedingt scheint, da Gesamtprotein und C $3 \mathrm{c}$ (Indikatoren der Blut-SynoviaSchranke) vergleichsweise niedrig ausfielen. $\mathrm{Da} \mathrm{a}_{2} \mathrm{M}$ in oberflächlichen Knorpelschichten gespeichert werden kann, könnte es bei der Knorpeldegeneration vermehrt in die Synovialflüssigkeit gelangen (Abb. 1 a).

\footnotetext{
* dankenswerterweise im Labor PD Dr. Müller/Dr. Ponz, MaxPlanck-Institut für Biochemie, Martinsried (b. München), durchgerührt.
}

Tab. 3 Biochemische Nachweismethode von Parametern in der Synoviadiagnostik

\begin{tabular}{|c|c|}
\hline Biochemische Parameter & Nachweismethoden \\
\hline $\begin{array}{l}\text { Glukose, Laktat, LDH, alkalische } \\
u \text {. saure Phosphatase. Lysozym. } \\
a_{2} \text {-Makroglobulin }\left(a_{2} M\right)\end{array}$ & Standard-Nachweisvertahren \\
\hline $\begin{array}{l}\text { Albumin. } C 3 c, a_{2} M_{1} \\
a_{1}-\text { Antitrypsin }\left(a_{1} A T\right)\end{array}$ & $\begin{array}{l}\text { Radiale Immundiffusion } \\
\text { (RID-Methode n Mancini und } \\
\text { Mitarb., 13) }\end{array}$ \\
\hline Albumın, $a_{1} P I$ & $\begin{array}{l}\text { Immun-Elektrophorese } \\
\text { (Rocket-Methode n. Laurell; 12) }\end{array}$ \\
\hline $\begin{array}{l}\text { lösliche. proteınhaltige } \\
\text { Knorpelabbauprodukte. } \\
\text { Albumin }\end{array}$ & $\begin{array}{l}\text { Diskontinuierliche } \\
\text { SDS-Elektrophorese } \\
\text { (Disk-Elektrophorese n. } \\
\text { Laemmli; 11) }\end{array}$ \\
\hline PMN-Elastase $\left(E-a_{1} P I\right)$ & $\begin{array}{l}\text { PMN-Elastase-Immunoassay } \\
\text { (n. Neumann und Mitarb. 14) }\end{array}$ \\
\hline
\end{tabular}

Eine mehr als 3 Jahre alte Meniskopathie mit Degenerationszeichen und akut-entzündlicher Reaktivierung, zeigte stark erhöhte Elastase- und Gesamtproteinwerte, die sonst nur noch in sehr zellreichen postoperativen Reizergüssen gefunden wurden. Bemerkenswert bei diesem Punktat ist auch der Nachweis löslicher proteinhaltiger Knorpelabbauprodukte (Abb. 1 a).

Frische traumatische Meniskus- und Kapsel-BandLäsionen zeigten eine stark erhöhte alkalische Phosphatasenaktivität, die auf erythrozytäre Zellelemente zurückzuführen ist (Abb. 1 b).

Alle postoperativen Reizergüsse boten einen verhältnismäßig höheren Wert für $\mathrm{C} 3 \mathrm{c}$ als für Gesamtprotein (C3c-Protein-Quotient [mg \%/g\%] > 10). Da in dieser Gruppe sowohl sehr zellarme als auch sehr zellreiche Punktate vertreten waren, erfolgte nachträglich eine Unterteilung in schwach und stark entzündliche Formen (Abb. 1 c).

In der Verlaufsbeobachtung zellarmer und zellreicher Formen postoperativer Reizergüsse zeigte sich, daß Proteinaseninhibitoren $\left(a_{2} M, a_{1} P I\right)$, Komplementspaltprodukte $(\mathrm{C} 3 \mathrm{c})$ und Gesamtprotein eine Erhöhung der Leukozytenzahl um das Mehrfache des Ausgangswertes nicht mit einer entsprechenden Konzentrationsänderung beantworten (Tab. 4).

Als einziger biochemischer Parameter zeigte die PMN-Elastase $\left(E-a_{1}\right.$ PI) im Vergleich zur Zellzahlerhöhung eine prozentual stärkere Konzentrationszunahme. Damit qualifiziert sich dieser Parameter, mit einer momentanen unteren Nachweisgrenze von $20 \mathrm{ng} / \mathrm{ml}$, als Gradmesser einer Entzündung.

Mit der Disk-Elektrophorese (Abb. 2 und 3) ließen sich - bis auf eine Ausnahme - keine langlebigen Kollagenbruchstücke oder sonstigen Abbauprodukte der Knorpelmatrix in der Synovialflüssigkeit nachweisen (Abb. 3).

Das Punktat mit einem positiven Ergebnis in der Disk-Elektrophorese, stammte von einem Patienten mit einer alten Meniskusläsion, die seit mehr als 3 Jahren zu Einklemmungserscheinungen und rezidivierenden Ergüssen führte: intraoperativ fand sich wenige Tage nach Punktion ein basisnah abgerissenes Innen- 


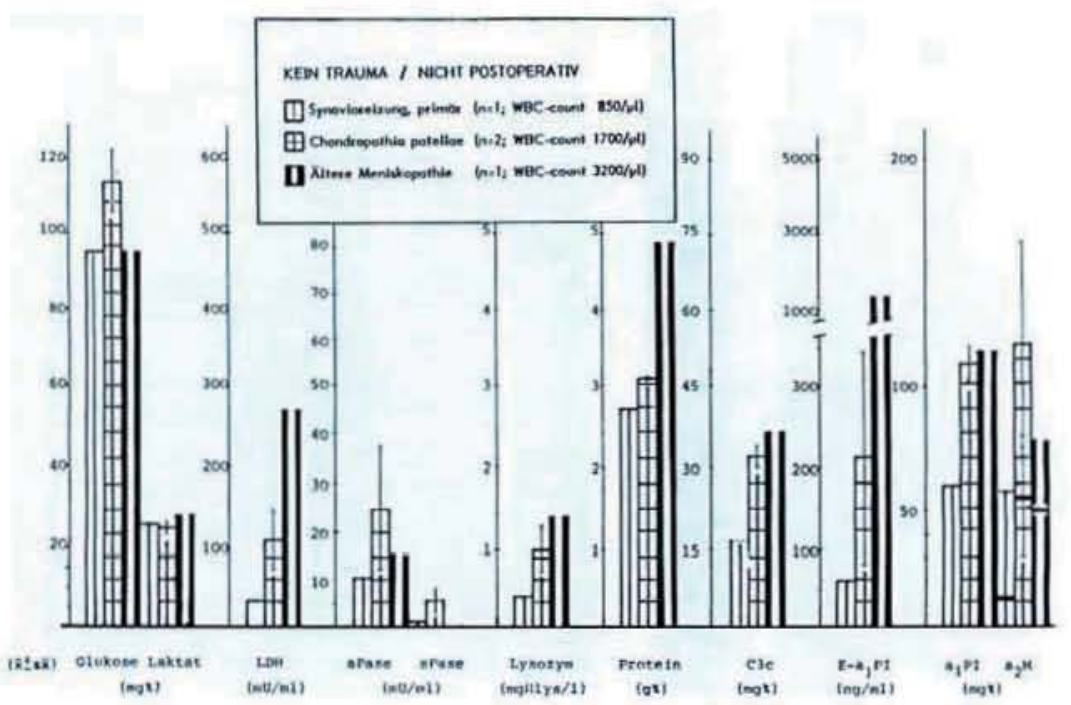

Abb. 1 a

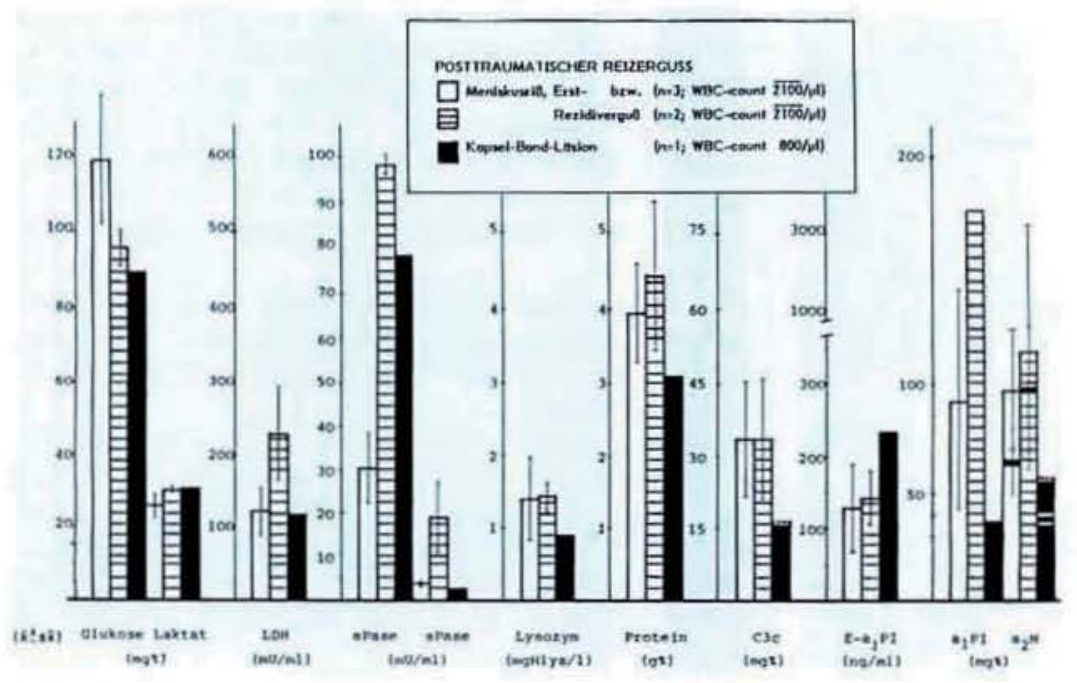

Abb. 1 b

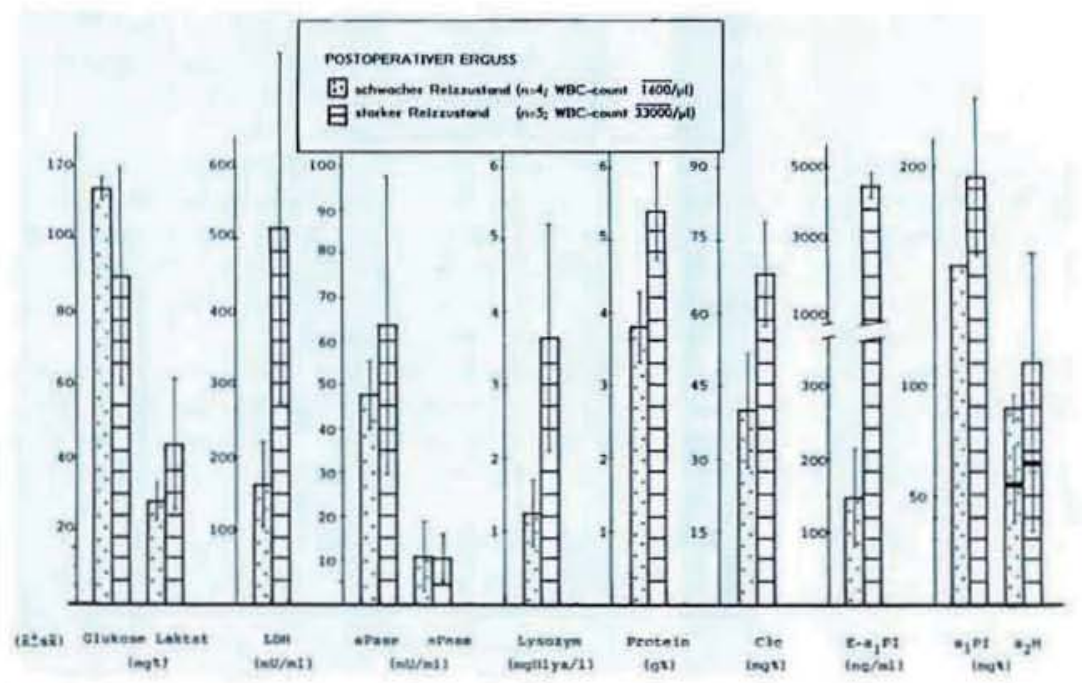

Abb.1a-c Ergebnisse der biochemischen Untersuchung der Synovialfiùssig. keit von Patienten mit primärer Synoviareizung. Chondropathia patellae, Meniskopathie (1 a) sowie posttraumatischen ( $1 \mathrm{~b}$ ) und postoperativen (1 c) Reizergüssen am Knie. gelenk.

Punktate von Patienten mit Chondropathia patellae und primarer Synoviareizung wiesen die geringsten Konzentrationen der gemessenen Parameter aut, frische Meniskus- oder Kapsel-Band-Lasionen zeigten eine stark erhohte alkalische Phosphataseaktivităt, wahrend postoperative Reizergüsse eine im Vergleich zu Gesamtprotein verhaltnismäig hohe $\mathrm{C} 3 \mathrm{c}$-Konzentration autwiesen. Bei Vorliegen zelireicher Punktate wie bei alterer Meniskopathie und postoperativen Reizergüssen mit starkem Reizzustand erreichte die PMN-Elastase (E-a,PI) die Hochstwerte dieser Untersuchung aP ase $=$ alkalische Phosphatase sP ase $=$ saure Phosphatase $a_{1} P I=a_{1}-P r o t e i n a s e n i n h i b i t o r$ Abb. 1c $a_{2} M=a_{2}-$ Mikroglobulin 


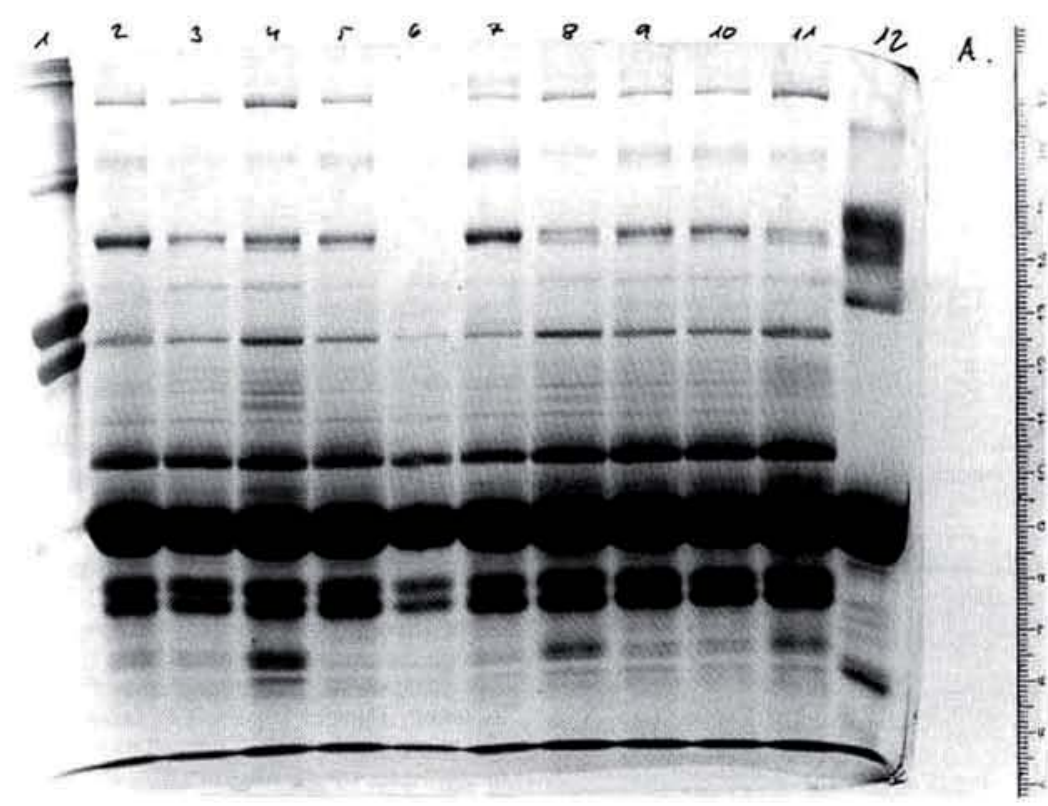

Abb. 2 Disk-elektrophoretische Auftrennung der Proteine pathologischer Synovialproben (Spalten 2-11). gegenübergestellt LRCS-Kollagen (Spalte 1) und Rinder-Albumin (Spalte 12). Durch Vergleich mit den Kollagen- und Albuminbanden (Albuminhauptbande: Ende des unteren Spaltendrittels) kann die Bandenintensität und -breite in den Synovialproben zur Objektivierung biochmisch ermittelter Albumin- und Gesamiproteinkonzentrationen sowie zur Identifizierung knorpelspezifischer Banden herangezogen werden.

Tab. 4 Verlaufsbeobachtung von 3 postoperativen Reizergüssen (Zustand nach Meniskektomie). Angegeben sind die Ausgangswerte (1. Untersuchung) für die Leukozytenzahl und die Konzentration bzw. Aktivität der biochemischen Parameter sowie deren Veränderung innerhalb von 14 Tagen (2. Untersuchung).

Die PMN-Elastase erlährt im Vergleich zur Leukozytenzahl und den übrigen biochemischen Parametern die stärkste Veränderung, während Proteinaseninhibitoren $\left(\mathrm{a}_{2} \mathrm{M}, \mathrm{a}_{1} \mathrm{Pl}\right)$ sowie Gesamtprotein und Komplementspaltprodukte nahezu unverändert blieben.

P. ase $=$ Phosphatase

$\mathrm{LDH}=$ Laktatdehydrogenase

\begin{tabular}{|c|c|c|c|c|c|c|c|c|c|c|c|c|c|c|c|c|c|c|}
\hline \multirow[b]{2}{*}{ 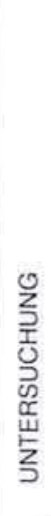 } & \multirow[b]{2}{*}{ 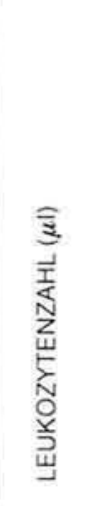 } & \multicolumn{17}{|c|}{ BIOCHEMISCHE PUNKTATUNTERSUCHUNG } \\
\hline & & 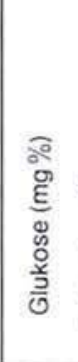 & 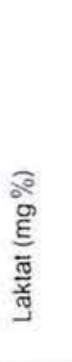 & 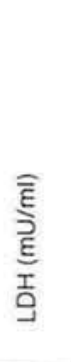 & 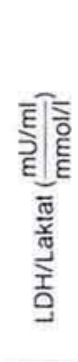 & 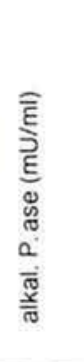 & 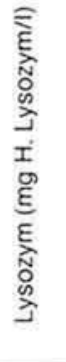 & 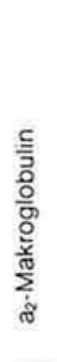 & 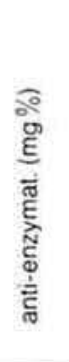 & 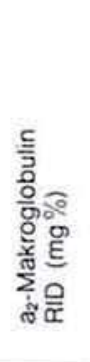 & 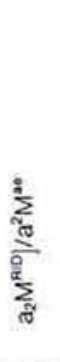 & 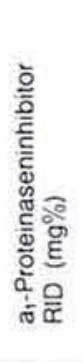 & 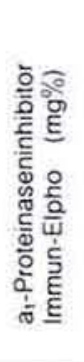 & 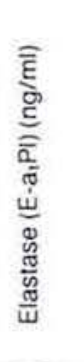 & 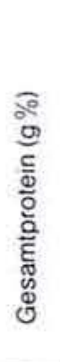 & 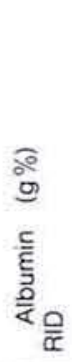 & 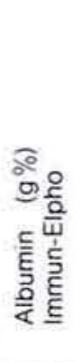 & 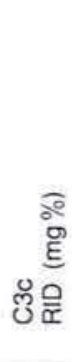 \\
\hline 1. & 2200 & 113.4 & 32.6 & 226 & 49 & 39,2 & 9.3 & 0.9 & 53.0 & 86.9 & 1,6 & 156.1 & 170,9 & 200 & 3.5 & 3.2 & 3,16 & 30.3 \\
\hline 2. & - & 116,7 & 32,6 & 178 & 49 & 49,0 & 9,1 & 1,6 & 73.8 & 97.0 & 1,3 & 156.1 & 150.5 & 205 & 4.5 & 3.7 & 2,58 & 39,5 \\
\hline 1. & 17000 & 115,5 & 32,1 & 461 & 129 & 53.7 & 8.6 & 3.2 & 32,1 & 67.6 & 2.1 & 156.1 & - & 4650 & 5.1 & 3,2 & - & 61,7 \\
\hline 2. & 72000 & 64,5 & 72,3 & 843 & 105 & 123 & 13,2 & 5.0 & 31.0 & 67.4 & 2,2 & 166.8 & 205.0 & 6300 & 6.3 & 2.9 & 3.54 & 82.0 \\
\hline 1. & 19500 & 74.7 & 33.7 & 506 & 135 & 34,8 & 7.5 & 3.2 & 88.8 & 186.1 & 2.1 & 247.3 & 402.9 & 2370 & 5.3 & 2.4 & 3.48 & 70.5 \\
\hline 2. & 53000 & 64.5 & 46,9 & 583 & 112 & 61.9 & 18,4 & 5.4 & 98.4 & 139.7 & 1.4 & 200.2 & 302.4 & 8760 & 5.8 & 2,6 & 3.64 & 66.8 \\
\hline
\end{tabular}

meniskushinterhorn sowie ein aufgefaserter Innenmeniskusrest mit massiven Degenerationszeichen. Die gefundenen löslichen kollagenasesensiblen Abbaupro- dukte könnten eine vorliegende Knorpelschädigung, beispielsweise an der medialen Femurkondyle, widerspiegeln (Abb. 3). 


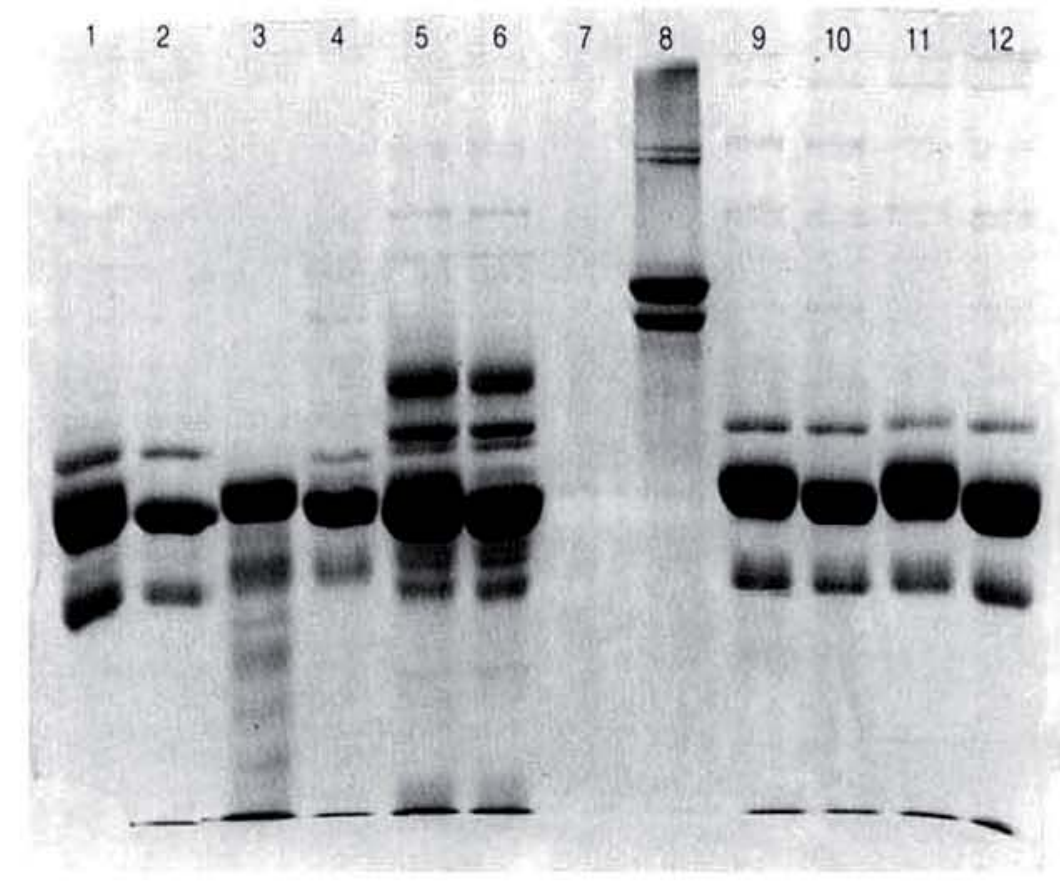

Abb.3 Disk-elektrophoretische Auftrennung der Proteine pathologischer Synovial. proben vom Menschen (Spalte 1-4) sowie normaler Synovialproben vom Plerd (Spal. te $5+6$ ), Rind (Spalte $9+10$ ) und Schwein (Spalte $11+12$ ) im Vergleich zu LRCS-Kollagen (Spalte $7+8$ ), einmal mit (ungerad. zahlige Spalten) und einmal ohne (geradzahlige Spalten) Kollagenasebehandlung. In einem Punktat (Spalte 3,4) ließen sich losliche proteinhaltige Knorpelbestandteile nachweisen, da es wie bei LRSC-Kollagen (Spalte 7.8) nach Kollagenasebehandlung zu einer Bandenauslöschung kam (vergleiche obere Halitte der Spalten 3 und 7).

\section{Literaturverzeichnis}

1) Akeson. W.H., Gershuni, D. H.: Articular cartilage and metabolism in health and disease. In: Resnick, D., G. Niwayama: Diagnosis of bone and joint disorders. Saunders Company, Philadelphia-London-Toronto (1981) 175-196

2) Binzus, G.: Substrate, Enzyme und Metabolite in der Synovialflüssigkeit verschiedener Genese. In: Synovialnüssigkeit und synoviales Milieu. Symposium Baden (bei Wien) 1977: Thumb, N., G. Kellner, G. Klein, H.Zeidler (Hrsg.): Thieme Verlag, Stuttgart (1979) 54-63

3) Dingerkus, M. L.: Möglichkeiten der Synoviadiagnostik zur Erfassung äthiologisch und prognostisch aussagekräftiger Parameter. Eine Untersuchung anhand von posttraumatischen, postoperativen und primär chondrodegenerativ bedingten Reizergüssen am Kniegelenk. Dissertationsarbeit der Technischen Universität München, eingereicht im März 1985

4) Gadek. J.E., Fells. G.A.. Wright D. G.. Crystal. R. G.: Human neutrophil elastase functions as a type III collagen "Collagenase". Biochemical and Biophysical Research Communications 95 (1980) 1815-1822

5) Greiling. H.. Kisters, R., Engels, G.: Die Enzyme in der Synovialflüssigkeit und ihre pathophysiologische Bedeutung. Enzymologia 30 (1965) 135-146

6) Greiling, H., Kleesiek, K., Stuhlsatz, H.W.: Zur klinischen Biochemie der Synovialnüssigkeit. In: Synovialflüssigkeit und synoviales Milieu. Symposium Baden (bei Wien) 1977. Thumb, N., Kellner, G., Klein, G., Zeidler, H. (Hrsg.) Thieme Verlag, Stuttgart (1979) 42-54

7) Jochum, M.. Duswald. K.H., Dittmer. H., Fritz. H.: Granulozytäre Elastase als lysosomales Markerenzym für pathobiochemische Veränderungen bei entzündlichen Erkrankungen. Berichte der ÖGKC 7 (1984) 53-59

8) Kleesiek. K.. Neumann.S.. Greiling. H.: Determination of the elastase-a - proteinase-inhibitor-complex, elastase activity and proteinase inhibitors in the synovial fluid. Fresenius Z. Anal. Chem. 311 (1982) 434-435

9) Kleesiek, K.. Brackertz. D.. Greiling. H.: Pathobiochemische Mechanismen bei chronisch-entzundichen Gelenkerkrankun- gen. In: Pathobiochemie der Entzündung. Lang, H., Greiling, H. (Hrsg.) Springer Verlag, Heidelberg-New York-Tokyo (1984) 203-225

10) Kuettner, K.E., Eisenstein, R., Sorgente, N.: Lysozyme of cartilage other connective tissue. In: Lysozyme. Ossermann, E. F. Canfield, R. E., Beychock, S. (Hrsg.) Academic (1974) 399-410 New York-London

11) Laemmli, U.K.: Cleavage of structural proteins during the assembly of the head of bacteriophage T4. Nature 227 (1970) 680-685

12) Laurell. C.B. (Hrsg.): Electrophoretic and electroimmunochemical analysis of proteins. The Scandinavian Journal of Clinical and Laboratory investigation, 29. Suppl. 124, Malmō, Nordens Boktrychere (1972) 21-37

13) Mancini, G.. Vaermann, J.P., Carbonara, A.O.. Heremans. J. F.: Single radial diffusionmethod for immunological quantitation of proteins. Protides Biol. Fluids 11 (1964) 370

14) Neumann, S., Hennrich, N., Gunzer, G., Lang. H.: Enzymelinked immunoassay for elastase from leukozytes in human plasma. In: J. Clin. Chem. Clin. Biochem. 22 (1984) 693-697

15) Otte, P.: Synovia und synoviales Milieu im Konzept der aktivierten Arthrose. In: Synovialflüssigkeit und synoviales Milieu. Symposium Baden (bei Wien) 1977. Thumb, N., Kellner, G., Klein, G., Zeidler, H. (Hrsg.) Thieme Verlag, Stuttgart (1979), 3-22

16) Rother. U.. Rother, K.: Komplement und zelluläre Interaktion. In: Pathobiochemie der Entzündung. Lang. $\mathrm{H}$., Greiling, $\mathrm{H}$ (Hrsg.) Springer Verlag. Heidelberg-New York-Tokyo (1984) $26-46$

17) Scherak, O., Weissel, M., Kolbe, H., Fritzsche, H., Kolarz, G.: $\beta_{2}$-Mikroglobulin in der Synovialflüssigkeit von Patienten mit chronischer Polyarthritis. In: Synovialflüssigkeit und synoviales Milieu. Symposium Baden (bei Wien) 1977. Thumb, N., Kellner, G., Klein, G., Zeidler., H. (Hrsg.). Thieme Verlag. Stuttgan (1979) 67-69

18) Thumb. N.. Kellner, G., Klein, G.. Zeidler, H.: Synovialnüssigkeit und synoviales Milieu. Symposium Baden (bei Wien) 1977. Thieme Verlag Stuttgart (1979)

Dr. M. L. Dingerkus

Poliklinik für Sportverletzungen

Connollystraße 32

8000 München 40 